\title{
Creating space for Indigenous perspectives on access and benefit-sharing: encouraging researcher use of the Local Contexts Notices
}

\author{
Libby Liggins $^{1}$, Maui Hudson ${ }^{2}$, and Jane Anderson ${ }^{3}$ \\ ${ }^{1}$ Massey University Auckland \\ ${ }^{2}$ University of Waikato \\ ${ }^{3}$ New York University
}

February 25, 2021

\begin{abstract}
A recent Molecular Ecology editorial made a proactive statement of support for the "Nagoya Protocol" and the principle of benefit-sharing (Marden et al. 2020) by requiring authors to provide a "Data Accessibility and Benefit-Sharing Statement" in their articles. Here, we encourage another step that enables Indigenous communities to provide their own definitions and aspirations for access and benefit-sharing alongside the author's "Statement". We invite the Molecular Ecology research community to use Biocultural-, Traditional Knowledge-, and Cultural Institution Notices to help Indigenous communities gain visibility within our research structures. Notices are one of the tools offered by the Biocultural Labels Initiative (part of the Local Contexts system) designed specifically for researchers and institutions. The Notices are highly visible, machine-readable icons that signal the Indigenous provenance of genetic resources, and rights of Indigenous communities to define the future use of genetic resources and derived benefits. The Notices invite collaboration with Indigenous communities and create spaces within our research systems for them to define the provenance, protocols, and permissions associated with genetic resources using Labels. Authors contributing to Molecular Ecology can apply Notices to their articles by providing the persistent unique identifier and an optional use-statement associated with the Notice in their "Data Accessibility and Benefit-Sharing Statement". In this way, our research community has an opportunity to accelerate support for the principles of the Nagoya Protocol, to alleviate concerns regarding Indigenous Data Sovereignty and equitable outcomes, and to build better relationships with Indigenous collaborators to enhance research, biodiversity, and conservation outcomes.
\end{abstract}

\section{Introduction}

In a recent editorial, the editors of Molecular Ecology and Molecular Ecology Resources made a timely and proactive statement of support for the "Nagoya Protocol" and the principle of benefit-sharing (Marden et al., 2020; and reiterated in Rieseberg et al., 2021). The 2014 "Nagoya Protocol on Access to Genetic Resources and the Fair and Equitable Sharing of Benefits Arising from their Utilization" is an international agreement to ensure that the benefits arising from the utilization of genetic resources are shared equitably (United Nations, 2011; https://www.cbd.int/abs/). The journals now encourage authors to disclose benefits generated, requesting an affirmative "Data Accessibility and Benefit-Sharing Statement" during the submission process that will be published alongside accepted articles (see: https://onlinelibrary.wiley.com/page/ journal/1365294x/homepage/forauthors.html; Marden et al., 2020). Such acknowledgement and advocacy of Indigenous rights and interests in biodiversity and genetic resources once again affirms Molecular Ecology and Molecular Ecology Resourcesas leaders seeking the improvement of research ethics and best practice in the fields of ecology and evolution.

In this Comment, we extend this advocacy by encouraging another step that enables Indigenous community's 
to provide their own definitions and aspirations for access and benefit-sharing (ABS) alongside the author's "Data Accessibility and Benefit-Sharing Statement". We contend that providing an Indigenous perspective on ABS is commensurate with the Nagoya Protocol and would benefit Indigenous communities, researchers, and will generate better biodiversity and conservation outcomes. Accordingly, we introduce the Biocultural (BC) Label Initiative - an agile system to help Indigenous communities gain visibility within our research structures. As part of this Initiative, we invite the research community to use Biocultural (BC), Traditional Knowledge (TK), and Cultural Institution (CI) Notices to create spaces for Indigenous perspectives within digital infrastructures and data repositories.

\section{Biocultural Labels and Notices}

The use of Biocultural (BC) Labels and Notices supports transparency and integrity in research engagements with Indigenous communities (e.g. tribes, clans; Anderson \& Hudson, 2020). The initiative is part of the Local Contexts system (www.localcontexts.org, Anderson \& Christen, 2019) that has successfully instilled information regarding the Indigenous provenance, context, and appropriate use of collections into digital infrastructures, through the use of Traditional Knowledge (TK) Labels and Notices (Anderson, 2012). In response to the Nagoya Protocol and concerns over Indigenous (genomic) Data Sovereignty (Carroll et al., 2020; Hudson et al., 2020), the BC Labels/Notices extend the inclusion of Indigenous interests to biological research (Anderson \& Hudson, 2020). The Labels and Notices (Figure 1) are designed to provide a highly visible (and in time, quickly recognizable), machine-readable, persistent and durable connection between collaborating Indigenous communities and: researchers, research projects and activity; the collected genetic resources, generated Digital Sequence Information (DSI), and knowledge; that exist as metadata in sample/data repositories and can appear on published articles.

The BC Labels support Nagoya Protocol expectations around the disclosure and origins of genetic resources (i.e. Provenance Label) and help to define and communicate Indigenous community expectations and consent about appropriate and future use of genetic resources and derived benefits. Importantly, BC Labels may only be applied by an Indigenous community and although the icons are generic in appearance, each Label has a persistent unique identifier and the Label metadata (as text) is customized to each use-case (potentially a research project, curated collection, a specimen, or collaboration). These community customized Labels are hosted on the Local Contexts Hub (https://localcontexts.org/tk-label-hub) and offer an Indigenous narrative regarding ABS that is not pre- or over-determined by researchers.

\section{Creating spaces for Indigenous perspectives}

The necessary augmentation of current research norms and infrastructures to acknowledge Indigenous interests will take time, but the most expedient transition will be achieved if all parties are empowered to enact change. Currently, over 60 Indigenous communities across 6 nations/countries are customizing Labels for multiple research programs, the Genomic Observatories Metadatabase (GEOME; Deck et al., 2017) has developed metadata fields supporting the interoperability of the initiative (Riginos et al., 2020), and several digital publishers have incorporated the Labels to make clear and visible Indigenous protocols for accessing and sharing knowledge (e.g. Scalar, https://scalar.me/anvc/scalar/; RavenSpace, https://ravenspacepublishing.org/). As a Molecular Ecology research community, we also have an opportunity to accelerate support for our Indigenous collaborators and the principles of the Nagoya Protocol through the use of the Notices - one of the Local Contexts tools specifically developed for researchers.

The Notices (Figure 1) are applied by researchers and/or institutions to support the recognition of Indigenous interests in collections and data. In particular, the Biocultural (BC) Notice and Traditional Knowledge (TK) Notice allow researchers to publicly acknowledge Indigenous rights and interests and pledge their commitment to meeting ABS principles. The TK Notice is used to signal that place-based knowledge carries accompanying cultural rights and responsibilities, meaning that appropriate permissions may need to be sought for future use of that knowledge; and the BC Notice signals the right of Indigenous communities to define the use of information, collections, and data (including DSI) generated from biodiversity and genetic resources associated with their traditional lands or waters. Unlike the Labels developed by communities, 
Notices are not customizable. Instead they are designed to be placeholders for the Labels when these have been generated by Indigenous communities. In this sense, the Notices initiate an equitable pathway within data systems for the inclusion of Indigenous rights and interests. Moreover, the use of Notices by researchers invites collaboration with Indigenous communities, normalizes use of the Local Contexts system, and creates physical spaces (e.g. appearing on publications and websites) and digital spaces (e.g. as metadata in repositories) for Indigenous provenance, protocols, and permissions.

To further activate and extend the inclusion of Indigenous interests into our research system, institutions and data repositories that hold collections of Indigenous origin can use Cultural Institution (CI) Notices. The 'Attribution Incomplete' Notice recognizes that collections and/or data have incomplete, inaccurate, and/or missing attribution. In identifying this missing attribution, there is an accompanying invitation for appropriate Indigenous communities to correct this exclusion or omission. The 'Open to Collaborate' Notice indicates that an institution is committed to developing new modes of collaboration, engagement, and partnership over collections. Together the CI Notices signal the commitment of an institution or repository to addressing missing information regarding the origins of specimens and genetic resources, and correspondingly their Indigenous contexts. As a practical mechanism, the Notices push towards standards for the inclusion of this information at a researcher and repository level. This increases capacity for meeting international obligations under the Nagoya Protocol.

Notices can be applied quickly and at any stage of a research program, including on data that is already published and deposited in publicly accessible repositories (such as provided by the International Nucleotide Sequence Database Collaboration; Cochrane, Karsch-Mizrachi, Takagi, \& Sequence Database Collaboration, 2016). To apply a Notice, researchers register with the Local Contexts Hub and create a profile using their ORCID iD (https://orcid.org/). The Hub will generate the Notice selected by the researcher and will send a notification to the relevant Indigenous community. Authors contributing to Molecular Ecology and Molecular Ecology Resources can apply Notices to their articles by providing the persistent unique identifier and an optional use-statement associated with the Notice in their "Data Accessibility and BenefitSharing Statement". Notices can also be connected to publications, specimens, DSI and derived genetic data held in other repositories through fields provided in GEOME (e.g. the 'TraditionalKnowledgeNotice' field used by the Ira Moana Project, https://sites.massey.ac.nz/iramoana/, and the 'Diversity of the Indo-Pacific Network', http://diversityindopacific.net/).

Local Contexts is currently focused on scaling these practical mechanisms for connecting Indigenous peoples with research and data collected on Indigenous lands and waters. There has been active uptake of the system by Indigenous communities, institutions and researchers in the US, Canada, Australia, Aotearoa New Zealand, Chile, and Spain. An international 'Cultural Institution Working Group' has been formed where experiences in using and implementing the Notices can be shared. The 'Aotearoa Biocultural Label Working Group' is also trialing the use of Notices and Labels across a diversity of research programs conducted at a national scale. The goal is to digitally integrate Indigenous rights and interests into research systems - in both research practice and in data storage and transfer - to alleviate concerns regarding Indigenous Data Sovereignty and ABS pertaining to genetic resources.

\section{The benefits of a research system that is inclusive of Indigenous perspectives}

The Biocultural (BC) Label Initiative is designed to directly support and benefit Indigenous communities, but it will also enhance research, biodiversity, and conservation outcomes. For instance, Traditional Knowledge often augments research (e.g. Ross et al., 2018), and the Notices and Labels provide a safe system to promote this exchange. The trust that is intrinsically built in the process of applying Labels will further facilitate meaningful future collaboration (discussed in Hudson et al., 2018, 2020), and potentially easier implementation of conservation and management plans informed by the research. Such a process enables Indigenous communities to be directly connected with research findings, to inform stewardship of resources according to traditional roles (Smith, 2016; Hudson et al., 2020), to act as citizen scientists, and to build capacity across diverse communities (Hudson et al., 2018; Nanibaa' et al., 2019). 
There are evident benefits academically and reputationally for researchers that use Notices. Engaging with the Local Contexts system provides researchers with a visible profile on the Local Contexts Hub promoting their research activity among researchers and Indigenous communities. The collaboration between Local Contexts and ORCiD will further support the recognition of researchers using Notices and will facilitate their transmission to publications. The application of Notices (and subsequently Labels) also tracks ABS beyond the initial collaboration, attributing greater impact to research and publications. Such transparency and accounting can be useful in our own peer-review research system. The Nagoya Protocol calls for domestic legislation to create legal guidelines for those seeking to access and utilize genetic resources for research and development (United Nations, 2011). The Biocultural Label Initiative is not proposed to replace domestic legislation, but can initiate a common standard for the research community as well as Indigenous communities collaborating across national boundaries, and for reviewers, editors, funding agencies, and downstream datausers wanting to support the principles of ABS.

As a research community there is incentive to support Indigenous interests and ABS (Eds., 2020 Nature Reviews Genetics ; Marden et al., 2020). Without appropriate means to build trust and safeguard Indigenous interests, research and most importantly, biodiversity and conservation outcomes for our shared futures may be compromised (Marden 2018; Marden et al., 2020; Smyth, Macall, Phillips, \& de Beer, 2020). To ensure equitable understanding of the opportunities and risks associated with the access and use of genetic resources, both Indigenous communities and our research community would benefit from the heightened transparency, communication, and accounting afforded by the BC Labels and Notices. We all have a role and a responsibility to forward new initiatives that place equity at the center of research practice and digital infrastructures. For example: researchers, reviewers, editors, and journals will need to encourage uptake; data repositories will need to implement and enable retention of Labels and Notices as metadata that interoperate with the Local Contexts Hub; and funders will need to request specific statements regarding Indigenous Data Sovereignty and ABS within data management plans. Crucially, Indigenous communities and researchers will need to work together toward mutual understanding. For authors publishing in Molecular Ecology and Molecular Ecology Resources , the provisioning of a "Data Accessibility and Benefit-Sharing Statement" is a place to start, and through additionally using Notices, we can also make space for our Indigenous collaborators and partners to be properly recognized, attributed and included. This will build more trust in the research and data, better relationships in practice for the future and more equitable outcomes.

\section{Acknowledgements}

The authors thank Brad Sherman and Cynthia Riginos for encouragement to contribute this comment. Development of the Biocultural Label Initiative has been supported by Catalyst Seeding funds for the project 'Te Tuākiri o te Tāonga: Recognizing Indigenous Interests in Genetic Resources' provided by the New Zealand Ministry of Business, Innovation and Employment and administered by the Royal Society Te Apārangi (19-UOW-008-CSG to MH and JA), leveraging the existing Local Contexts (https://localcontexts.org/) platform supported by the National Endowment for the Humanities (PR 234372-16 and PE 263553-19 to JA) and the Institute of Museums and Library Services in the US (RE-246475-OLS-20 to JA), New York University Graduate School of Arts and Sciences, and the University of Waikato. Continuing infrastructure development is supported through ENRICH - Equity for Indigenous Research and Innovation Coordinating Hub based at New York University and University of Waikato (https://www.enrich-hub.org/). The Biocultural Label Initiative is extended through use-cases, supported and refined by the Aotearoa Biocultural Label Working Group, FOMA Innovation (https://www.foma.org.nz/), Te Mana Rauranga (https://www.temanararaunga.maori.nz/), Genomics Aotearoa (https://www.genomics-aotearoa.org.nz/), Indigenous Design and Innovation Aotearoa (https://www.idia.nz/), the Genomics Observatories Metadatabase (https://geome-db.org/), the Ira Moana - Genes of the Sea - Project (www.massey.ac.nz/iramoana, supported by Catalyst Seeding funds provided by the New Zealand Ministry of Business, Innovation and Employment and administered by the Royal Society Te Apārangi, 17-MAU-309-CSG to LL), and a Massey University Research Fund to LL.

\section{References}


Anderson, J. (2012). Options for the future protection of GRTKTCES: the traditional knowledge license and labels initiative. Journal of World Intellectual Property Organization, 4 (1), 66-75.

Anderson, J., \& Christen, K. (2019). Traditional knowledge (TK) Labels. Retrieved from https://localcontexts.org/ tk-labels/

Anderson, J., \& Hudson, M. (2020). The Biocultural Labels Initiative: Supporting Indigenous rights in data derived from genetic resources.Biodiversity Information Science and Standards, 4, e59230. doi: $10.3897 /$ biss. 4.59230

Carroll, S.R., Garba, I., Figueroa-Rodríguez, O.L., Holbrook, J., Lovett, R., Materechera, S., ... Sara, R. (2020). The CARE Principles for Indigenous Data Governance. Data Science Journal ,19 (1), 1-12. doi: $10.5334 /$ dsj-2020-043/

Cochrane, G., Karsch-Mizrachi, I., Takagi, T., \& Sequence Database Collaboration, I.N. (2016). The international nucleotide sequence database collaboration. Nucleic Acids Research, 44 (1), 48-50. doi: $10.1093 / \mathrm{nar} / \mathrm{gkv} 1323$

Deck, J., Gaither, M. R., Ewing, R., Bird, C. E., Davies, N., Meyer, .. Crandall, E. D. (2017). The Genomic Observatories Metadatabase (GeOMe): A new repository for field and sampling event metadata associated with genetic samples. PLoS Biology, 15 (8), e2002925. doi: 10.1371/journal.pbio.2002925

Editorial. (2020). Better together. Nature Reviews Genetics, 21 , 333. doi: 10.1038/s41576-020-0238-8

Hudson, M., Nanibaa'A, G., Sterling, R., Caron, N.R., Fox, K., Yracheta, J., .. Taualii, M., (2020). Rights, interests and expectations: Indigenous perspectives on unrestricted access to genomic data.Nature Reviews Genetics, 21 (6), 377-384. doi: 10.1038/s41576-020-0228-x

Hudson, M., Wilcox, P., Smith, B., Beaton, A., Milne, M., \& Russell, K. (2018). (C)ELSI-us: Reducing Friction with Indigenous Communities in Genomic Research. American Anthropologist, 120 (2), 330-332. doi: 10.1111/aman.13043

Marden, E. (2018). International agreements may impact genomic technologies. Nature Plants , 4, 2- 4. doi: $10.1038 / \mathrm{s} 41477-017-0087-4$

Marden, E., Abbott, R. J., Austerlitz, F., Ortiz-Barrientos, D., Baucom, R. S., Bongaerts, P., .. Rieseberg, L. H. (2020). Sharing and reporting benefits from biodiversity research. Molecular Ecology . doi: $10.1111 /$ mec. 15702

Nanibaa'A, G., Hudson, M., Ballantyne, L.L., Garba, I., Martinez, A., Taualii, M., .. Rainie, S.C. (2019). Genomic research through an indigenous lens: understanding the expectations. Annual Review of Genomics and Human Genetics, 20 , 495-517. doi: 10.1146/annurev-genom-083118-015434

Rieseberg, L., Warschefsky, E., O’Boyle, B., Taberlet, P., Ortiz-Barrientos, D., Kane, N. C., \& Sibbett, B. (2021). Editorial 2021. Molecular Ecology, 30, 1-25. doi: 10.1111/mec.15759

Riginos, C., Crandall, E.D., Liggins, L., Gaither, M.R., Ewing, R.B., Meyer, C., . . Deck, J. (2020). Building a global genomics observatory: Using GEOME (the Genomic Observatories Metadatabase) to expedite and improve deposition and retrieval of genetic data and metadata for biodiversity research. Molecular Ecology Resources, 20 (6), 1458-1469. doi: 10.1111/1755-0998.13269

Ross, P. M., Knox, M. A., Smith, S., Smith, H., Williams, J., \& Hogg, I. D. (2018). Historical translocations by Māori may explain the distribution and genetic structure of a threatened surf clam in Aotearoa (New Zealand). Scientific Reports, 8 , 17241. doi: 10.1038/s41598-018-35564-4

Smith, D. (2016). Governing data and data for governance: the everyday practice of Indigenous sovereignty. In Kukutai, T. \& Taylor, J. (Eds.), Indigenous data sovereignty: Toward an agenda . pp. 117-135. Canberra, Australia: Australian National University Press. doi: 10.22459/CAEPR38.11.2016.07 
Smyth, S. J., Macall, D. M., Phillips, P. W., \& de Beer, J. (2020). Implications of biological information digitization: Access and benefit sharing of plant genetic resources. The Journal of World Intellectual Property , 23 (3-4), 267-287. doi: 10.1111/jwip.12151

United Nations. (2011). Nagoya Protocol on Access to Genetic Resources and the Fair and Equitable Sharing of Benefits Arising from their Utilization to the Convention on Biological Diversity. Retrieved from https://www.cbd.int/abs/

\section{Data Accessibility and Benefit-Sharing Statement}

No genetic resources/data were generated or used in this comment, however it is intended to support greater benefit-sharing. This comment has benefitted from the input of several Indigenous communities.

\section{Author Contributions}

JA and MH designed the research, formed necessary collaborations, and secured the majority of the project funding, with input from LL and several other colleagues and collaborators. LL wrote the paper, with guidance and editorial help from JA and $\mathrm{MH}$.

\section{Tables and Figures}

Figure 1. An overview of the Local Contexts system, and the role of researchers and institutions in applying Notices. BC: Biocultural, TK: Traditional Knowledge, CI: Cultural Institution.

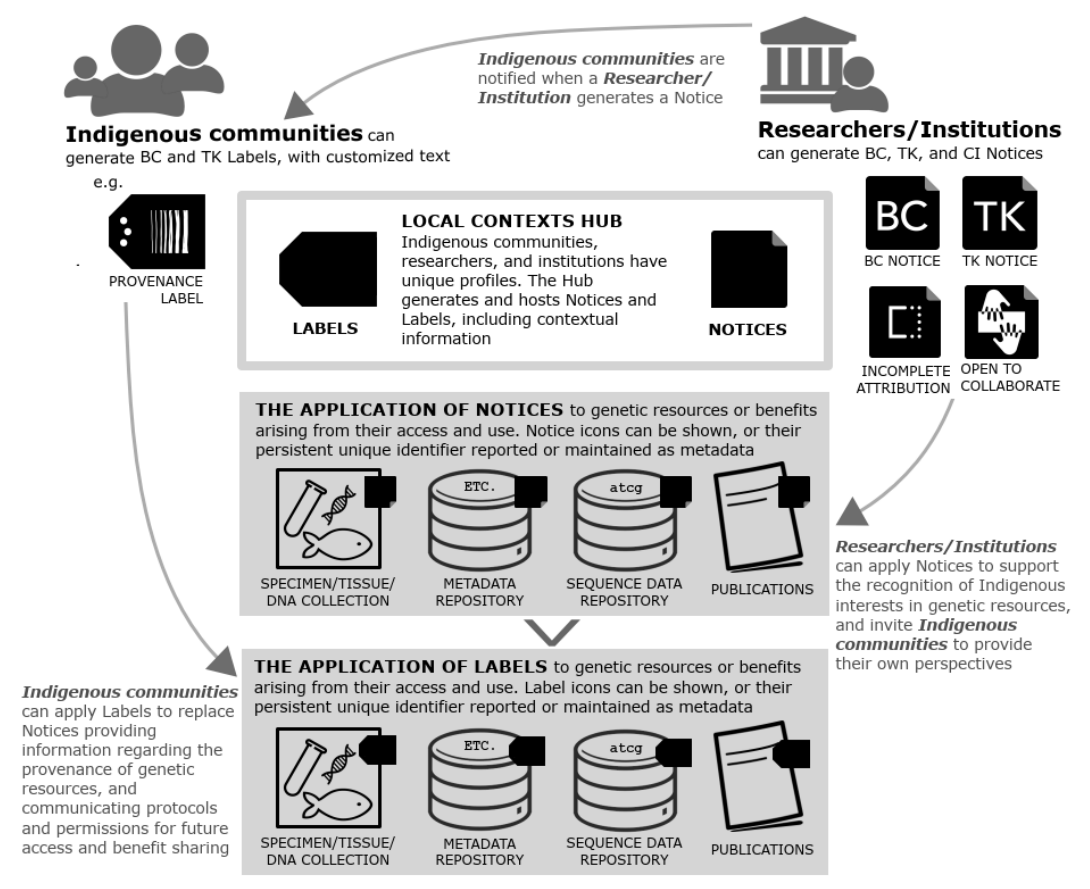

